\title{
HYPERGRAPHS WITH FINITELY MANY ISOMORPHISM SUBTYPES
}

\author{
HENRY A. KIERSTEAD AND PETER J. NYIKOS
}

\begin{abstract}
Let $\mathscr{H}=(H, E)$ be an $n$-uniform infinite hypergraph such that the number of isomorphism types of induced subgraphs of $\mathscr{H}$ of cardinality $\lambda$ is finite for some infinite $\lambda$. We solve a problem due independently to Jamison and Pouzet, by showing that there is a finite subset $K$ of $H$ such that the induced subgraph on $H-K$ is either empty or complete. We also characterize such hypergraphs in terms of finite (not necessarily uniform) hypergraphs.
\end{abstract}

In a 1981 colloquium lecture at the University of South Carolina, R. Jamison posed the following problem:

If an infinite $n$-uniform hypergraph $\mathbf{H}=(H, E)$ is isomorphic to each of its induced subgraphs of cardinality $|H|$, must $\mathbf{H}$ be either empty $(E=\varnothing)$ or complete $\left(E=[H]^{n}\right)$ ?

In other words, must $H$ be either independent or a clique? Recall that if $H^{\prime} \subset H$, then the subgraph $H\left\lceil H^{\prime}\right.$ induced on $H^{\prime}$ is $\left(H^{\prime}, E \cap\left[H^{\prime}\right]^{n}\right)$.

In the case of graphs $(n=2)$ and 3-uniform hypergraphs of regular cardinality, there are simple affirmative solutions, found by Jamison and the authors and (independently) by M. Pouzet. But already the case of 3-uniform hypergraphs of singular cardinality is difficult enough so that it remained unsolved until 1985, shortly before the general solution provided in this paper; moreover, neither of the first two proofs for $n=3$ by the authors seems to extend to $n=4$.

Our first theorem provides an affirmative answer to Jamison's problem under formally weaker hypotheses on $\mathbf{H}$. Three of our other theorems also imply an affirmative answer. Given a hypergraph $\mathbf{H}$, and induced subgraphs $\mathbf{H}_{1}$ and $\mathbf{H}_{2}$ of $\mathbf{H}$, we write $\mathbf{H}_{1} \simeq \mathbf{H}_{2}$ if $\mathbf{H}_{1}$ and $\mathbf{H}_{2}$ are isomorphic. This is obviously an equivalence relation, and if $\mu \leq|H|$ we let $\mathbf{I}_{\mu}(\mathbf{H})$ be the set of all isomorphism classes of induced subgraphs of cardinality $\mu$, and let $\mathbf{I}_{|H|}(\mathbf{H})$ be denoted $\mathbf{I}(\mathbf{H})$. Jamison also posed the problem: if $I(H)$ is finite, must $\mathbf{H}$ either have a clique or an independent subset of cardinality $|H|$ ? (His first question is essentially

Received by the editors February 3, 1986 and, in revised form, November 3, 1987.

1980 Mathematics Subject Classification (1985 Revision). Primary 05C65, 05C75; Secondary 03 C99.

Research by H.A.K. supported in part by the National Science Foundation under ISP-80110451 and the Office of Naval Research under N00014-85K-0494; research by P.J.N. supported in part by the National Science Foundation under MCS-8301916. 
the case $\mathbf{I}(\mathbf{H})=1$.) Our second theorem provides an affirmative answer, and we sharpen it to

Theorem 3. Let $\mathbf{H}=(H, E)$ be an infinite n-uniform hypergraph. If, for some infinite $\mu \leq|H|, \mathbf{I}_{\mu}(\mathbf{H})$ is finite, then there is a subset $K$ of $H$ such that $|K| \leq\left|\mathbf{I}_{\mu}(\mathbf{H})\right|-1$ and such that the induced subgraph on $H-K$ is either empty or complete.

Jamison's problems arose from the study of freedom in algebraic closure systems. An algebraic closure system $(X, L)$ is $(\alpha, N)$-homogeneous if there are $N$ isomorphism types of subspaces of cardinality $\alpha$. Let $M(\alpha, N)$ denote the smallest cardinal greater than or equal to $\alpha$ such that for any $n$, if $\mathbf{H}$ is an $n$-uniform hypergraph of cardinality at least $M(\alpha, N)$ and $\left|\mathbf{I}_{\alpha}(\mathbf{H})\right|=N$ then $\mathbf{H}$ contains a complete subgraph or an empty subgraph of cardinality $\alpha$. Jamison [3] proved that for each finite $N$, if $(X, L)$ is an $(\alpha, N)$-homogeneous algebraic closure system such that $M(\alpha, N)<|X|$ and either $N=1$ or, for some regular cardinal $\beta, M(\alpha, N-1) \leq \beta \leq|X|$, then there exists a subset $F \subset X$ with $|F|=N-1$ such that the subspace $F-X$ is $k$-free for some $k=0,1,2, \ldots, \infty$. He was able to provide a trivial upper bound on $M(\alpha, N)$ using infinite Ramsey theory. Our results show that $M(\alpha, N)=\alpha$.

Pouzet discovered these problems independently of Jamison. For general relational systems $R=\left(A, R_{i}\right)_{i \in I}$, he considered the function $\phi_{\mathbf{R}}(\mu)=\left|\mathbf{I}_{\mu}(\mathbf{R})\right|$. He proved, subject to a positive solution to the second problem, i.e., Theorem 2 , that if $\phi_{\mathbf{R}}(\mu)$ is finite for some infinite $\mu$, then $\phi_{\mathbf{R}}$ is increasing up to some integer $\mu_{0}$ and then either (i) $\phi_{\mathbf{R}}$ is constant on $\left[\mu_{0},|A|\right]$ or (ii) $\mu=|A|$ and $\phi_{\mathbf{R}}$ is constant on $\left[\mu_{0}, \omega\right)$ and $\phi_{\mathbf{R}}(\nu)=\nu^{+}$for $\nu \in[\omega,|A|)$ and $\phi_{\mathbf{R}}\left(\mu_{0}\right) \leq \phi_{\mathbf{R}}(\mu)$. The archetypal example of the latter behavior is provided by $\mathbf{R}=(\kappa, \leq)$ when $\kappa$ is a cardinal number. In the case of hypergraphs, only the former behavior is possible:

Theorem 4. Let $\mathbf{H}=(H, E)$ be an infinite $n$-uniform hypergraph. If, for some infinite $\mu \leq|H|, \mathbf{I}_{\mu}(\mathbf{H})$ is finite, then $\left|\mathbf{I}_{\lambda}(\mathbf{H})\right|=\left|\mathbf{I}_{\mu}(\mathbf{H})\right|$ for all infinite $\lambda \leq|H|$.

Of course, Ramsey's theorem on $\omega$, one version of which states that every countably infinite $n$-uniform hypergraph has either an infinite clique or an infinite independent subset, implies an affirmative solution to Jamison's original problem, given Theorem 4: let $\mu=|H|$ and $\lambda=\omega$ and $\left|\mathbf{I}_{\mu}(\mathbf{H})\right|=1$. But what is most revealing about Theorem 4 is the technique used in its proof. It sets up a natural 1-1 correspondence between $\mathbf{I}_{\mu}(\mathbf{H})$ and $\mathbf{I}_{\lambda}(\mathbf{H})$ which, in particular $(\lambda=|H|)$, allows us to recover the isomorphism type of $\mathbf{H}$ :

Theorem 5. If $\mathbf{I}_{\mu}(\mathbf{H})$ is finite for some infinite $\mu$, then the isomorphism type of $\mathbf{H}$ is determined by the isomorphism types of the induced subgraphs of $\mathbf{H}$ of cardinality $\mu$.

Theorem 6. If $\mathbf{I}_{\mu}(\mathbf{H})$ is finite for some infinite $\mu$, then the first-order theory of $\mathbf{H}$ is finitely axiomatizable and categorical in every infinite cardinal. 
The 1-1 correspondence is part of a general theory which we develop here and which allows us to reduce the entire theory of $n$-uniform hypergraphs with finite $\mathbf{I}(\mathbf{H})$ to the theory of (not necessarily uniform) hypergraphs with finitely many edges, each of size $\leq n$. To each infinite $n$-uniform hypergraph $\mathbf{H}$ we associate a hypergraph $\mathbf{S}(\mathbf{H})$ with the same underlying set, such that $\mathbf{S}(\mathbf{H})$ has finitely many edges if, and only if, $\mathbf{I}(\mathbf{H})$ is finite. The correspondence $\mathbf{S}$ is functorial on isomorphisms and, if $\mathbf{I}(\mathbf{H})$ is finite, on embeddings of infinite $n$-uniform hypergraphs into $\mathbf{H}$. Specifically, if $\mathbf{I}(\mathbf{H})$ is finite, and $f: H^{\prime} \rightarrow H$ is an injective function from the vertex set of an infinite $n$-uniform hypergraph $\mathbf{H}^{\prime}$, then $f$ is an embedding of $\mathbf{H}^{\prime}$ into $\mathbf{H}$ iff it is an embedding of $\mathbf{S}\left(\mathbf{H}^{\prime}\right)$ into $\mathbf{S}(\mathbf{H})$. Thus $\mathbf{I}_{\mu}(\mathbf{H})$ is in 1-1 correspondence with $\mathbf{I}_{\mu}(\mathbf{S}(\mathbf{H}))$ for all infinite $\mu$, and in the latter family we have only finitely many edges to deal with.

On being told of our first theorem, P. Erdös asked whether it extends to what might be called $\omega$-uniform hypergraphs: pairs $(H, E)$ where $E$ is a collection of $\omega$-element subsets of $H$ and induced subgraphs etc. are defined in the obvious way. A. Hajnal later observed the following counterexample: $H=\omega_{1}$ and $E$ is the collection of subsets of order type $\omega$.

Our second theorem may be looked upon as an attempt to extend Ramsey theory beyond the countable case: an $n$-uniform hypergraph must either have a clique of size $\kappa$, or an independent subset of size $\kappa$, or infinitely many pairwise nonisomorphic induced subgraphs of size $\kappa$. An interesting question is whether "infinitely many" can be improved, and to what extent, especially for regular $\kappa$.

Problem 1. Let $\kappa$ be a regular uncountable cardinal, $n \geq 2$. If an $n$-uniform hypergraph $\mathbf{H}$ of cardinality $\kappa$ has no clique or independent subset of cardinality $\kappa$, must $\mathbf{I}(\mathbf{H})$ be uncountable?

We have no affirmative solution to Problem 1 even in case $n=2$. [We do have an affirmative solution in case $\kappa=\omega_{1}, n=2$ under PFA, as will be shown in a future paper, but no "ZFC solution."] On the other hand, we have no negative solution for any $n$ or $\kappa$ to

Problem 2. Let $\kappa$ be a regular cardinal, $n \geq 2$. If an $n$-uniform hypergraph $\mathbf{H}$ of cardinality $\kappa$ has no clique or independent subset of cardinality $\kappa$, is $|\mathbf{I}(\mathbf{H})|=2^{\kappa}$ ?

In $\S 5$ we will see that the classical examples of graphs with no uncountable clique or independent set cannot serve as counterexamples to Problem 2.

Other cardinals besides $\omega_{1}$ and $2^{\kappa}$ can be reasonably considered as candidates for the minimal size of $\mathbf{I}(\mathbf{H}): \kappa, 2^{<\kappa}$, and $2^{\chi(\kappa)}$ where $\chi(\kappa)$ denotes the number of cardinals (finite or infinite) that precede $\kappa$. For singular $\kappa$, $\chi(\kappa)^{c f(\kappa)}$ seems to be the best candidate, in view of the example of the disjoint union of $c f(\kappa)$ cliques of size less than $\kappa$. But if we modify the question to ask how large $\mathbf{I}(\mathbf{H})$ must be if there is to be a $\lambda<\kappa$ such that $H$ has no clique 
or independent subset of size greater than $\lambda$, then all of the above cardinals could be reasonable candidates for a solution, as well as $c f(\kappa)$.

In $\S 1$ we mention another set of problems motivated by our first theorem: rather than asking how many isomorphism classes we need for a "non-Ramsey" example, we ask how few elements we can remove and still obtain nonisomorphic subgraphs. Other unsolved problems concerning $\mathbf{I}(\mathbf{H})$ are at the end of $\S 3$.

Most of our terminology and notation is standard. A hypergraph is a pair $\mathbf{H}=(H, E)$ where $H$ is a set of elements called vertices and $E$ is a (possibly empty) collection of finite (possibly empty) subsets of $H$, called the edges of $\mathbf{H}$. The order of $\mathbf{H}$ is the least upper bound on the cardinality of the edges of $\mathbf{H}$. An injective function $f: H \rightarrow H^{\prime}$ is an embedding of $(H, E)$ onto $\left(H^{\prime}, E^{\prime}\right)$ provided that $e \in E$ iff $f[e] \in E^{\prime}$, for all $e \subset H$. An isomorphism is a surjective embedding. A hypergraph $\left(H_{1}, E_{1}\right)$ is an induced subhypergraph of $(H, E)$ if $H_{1} \subset H$ and the insertion of $H_{1}$ into $H$ is an embedding. For the sake of brevity we write "induced subgraph" and frequently omit the word "induced."

As usual, given $n \in \omega,[H]^{n}$ denotes the set of $n$-element subsets of $H$. A hypergraph $\mathbf{H}=(H, E)$ is n-uniform if $E \subset[H]^{n} . \mathbf{H}$ is called empty if $E=\varnothing$, complete if $E=[H]^{n}$. Given $k \in \omega$, a $k$-element subset of $H$ is called a $k$-tuple. Vertices are designated by letters $x, y, z, w, v, u$ and $k$ tuples by the same letters with bars: $\bar{x}$, etc. Unions of $k$-tuples are designated by juxtaposition: $\bar{x} \bar{y}$ means $\bar{x} \cup \bar{y}, \bar{x} y$ means $\bar{x} \cup\{y\}$.

An $n$-uniform hypergraph can be thought of as a model with one $n$-ary predicate $R$ which is "fully symmetric," that is,

$$
\left(x_{1}, \ldots, x_{n}\right) \in R \quad \text { iff } \quad\left(x_{\sigma(1)}, \ldots, x_{\sigma(n)}\right) \in R
$$

for each permutation $\sigma$ of $\{1, \ldots, n\}$, and "fully antireflexive," that is, if $\left(x_{1}, \ldots, x_{n}\right) \in R$ then $x_{i} \neq x_{j}$, for all $i \neq j$. In this way, one can speak (cf. $\S \S 1-3$ and 1-4 of [2]) of the theory of $n$-uniform hypergraphs. An induced subgraph is then simply a submodel, and R. Jamison's first question refers to the following concept:

Definition 0. A model $A=(A, J)$ of a theory $T$ is smooth if it is isomorphic to each of its large submodels (viz. submodels $B$ whose underlying set $B$ has the same cardinality as $A$ ).

A hypergraph can be thought of as a concatenation, over various $n \in \omega$, of $n$-uniform hypergraphs sharing the same underlying set, and many statements about isomorphisms, smoothness, etc. are routinely reducible to statements about the individual $n$-uniform "parts." For example, from our affirmative solution of Jamison's first problem, it follows routinely that a hypergraph $(H, E)$ is smooth iff for all $n, E \cap[H]^{n} \neq \varnothing$ implies $[H]^{n} \subset E$. For the sake of simplicity, then, most of our results are stated in terms of $n$-uniform hypergraphs. 
The dual of an $n$-uniform hypergraph $(H, E)$ is $\left(H,[H]^{n}-E\right)$. Many concepts and statements have easily recognizable duals. Given a sentence $S$ in the theory of $n$-uniform hypergraphs, a sentence $S^{*}$ is dual to $S$ if $(H, E) \models S$ is equivalent to $\left(H,[H]^{n}-E\right) \models S^{*}$.

\section{THE TRIVIALITY OF SMOOTH HYPERGRAPHS}

The title result of this section was first shown in 1984 for hypergraphs of regular cardinality by the authors. Shortly thereafter, it was shown for hypergraphs of singular strong limit cardinality by J. Larson and R. Laver. The argument presented here is a subtle variation on the authors' original argument in the regular case. The Larson-Laver proof, as well as the proofs for $n=2$ and $n=3$ mentioned in the introduction, were quite different.

Because it only introduces minor complications, we actually show something more general than the result in the title.

Definition 1. An infinite hypergraph $\mathbf{H}=(H, E)$ is almost smooth if it is $n$ uniform, and isomorphic to $\mathbf{H}\left\lceil H^{\prime}\right.$ whenever $H^{\prime} \subset H$ and $\left|H-H^{\prime}\right|<|H|$. $\mathbf{H}$ is $\mu$-hereditarily almost smooth if every subgraph of cardinality $\mu$ is almost smooth.

Theorem 1. If $\mathbf{H}=(H, E)$ is $\mu$-hereditarily almost smooth for some infinite $\mu \leq|H|$, then either $E=\varnothing$ or $E=[H]^{n}$.

For $\mu=\omega$, Theorem 1 is a quick corollary of Ramsey's theorem: $H$ either has an infinite clique $H^{\prime}$ or an infinite independent subset. If $H$ is not a clique in the first case, let $\bar{x}$ be a nonedge of cardinality $n$. Then $H^{\prime} \cup \bar{x}$ is not isomorphic to $H^{\prime}$. The other case is similar.

Before proving Theorem 1, we introduce some concepts and auxiliary results. Throughout this section, $\mathbf{H}=(H, E)$ will denote an $n$-uniform hypergraph.

Definition 2. If $\bar{x}$ is an $n$-tuple in $H$, then $\bar{x}$ is almost universal (abbreviated a.u.) if $\bar{x} \in E$ and almost isolated (abbreviated a.i.) if $\bar{x} \notin E$. If $\bar{x}$ is a $k$-tuple, $0 \leq k<n$, then $\bar{x}$ is almost universal if $\mid\{y: \bar{x} y$ is a.u. $\} \mid>$ $\mid\{y: \bar{x} y$ is not a.u. $\} \mid$ and $\bar{x}$ is almost isolated if $\mid\{y: \bar{x} y$ is a.i. $\}|>|[y: \bar{x} y$ is not a.i. $\} \mid$. The valence of a $k$-tuple $\bar{x}, 0 \leq k<n$, is $v(x)=\mid\{y: \bar{x} y$ is a.u. $\} \mid$. The deficiency of an a.u. $k$-tuple $\bar{x}$ is $d(\bar{x})=\mid\{y: \bar{x} y$ is not a.u. $\} \mid$ and the deficiency of an a.i. $k$-tuple $\bar{x}$ is $d(\bar{x})=\mid\{y: \bar{x} y$ is not a.i. $\} \mid$.

Note that if $\bar{x}$ is almost universal in $\mathbf{H}, \bar{x} \in H^{\prime} \subset H$, and $\left|H^{\prime}\right|=H$, then $\bar{x}$ is almost universal in $\mathbf{H}^{\prime}$. The converse need not be true unless $\left|H-H^{\prime}\right|<|H|$. This complicates the proof of

Main Lemma. If $|H|=\kappa \geq \omega$, and $1 \leq k \leq n$, and $\lambda$ is any cardinal such that $\lambda \leq \kappa$ if $\kappa$ is regular and $\lambda<\kappa$ if $\kappa$ is singular, then there is a subgraph $\mathbf{H}(k, \lambda)$ of $\mathbf{H}$ such that $|\mathbf{H}(k, \lambda)|=\kappa$ and an ordering $\left\{x_{\alpha}: \alpha<\kappa\right\}$ of $H(k, \lambda)$ 
such that:

(*) If $\left\{x_{\xi(1)}, \ldots, x_{\xi(k)}\right\}$ is almost universal in $H(k, \lambda)$ and is listed in natural order $(\xi(i)<\xi(j)$ if $i<j)$, then $H(k, \lambda) \vDash v\left(x_{\xi(1)}, \ldots, x_{\xi(k-1)}\right) \geq$ $|\xi(k)|^{+} \cdot \lambda$. [Convention: $\left.\left\{x_{\xi(1)}, x_{\xi(0)}\right\}=\varnothing.\right]$

We will use the full force of this lemma in $\S 2$; here we will need only the fact that $H(k, \lambda) \vDash v\left(x_{\xi(1)}, \ldots, x_{\xi(k-1)}\right) \geq 2$. (The symbol $\vDash$ denotes satisfaction; in this last case, for example, it indicates that $\left\{x_{\xi(1)}, \ldots, x_{\xi(k-1)}\right\}$ extends to at least two $k$-tuples that are a.u. in $H(k, \lambda)$.)

Corollary 1. If $\mathbf{H}$ is $\kappa$-hereditarily almost smooth and $|H|=\kappa$, and $\mathbf{H}$ has an almost universal $k$-tuple, $1 \leq k \leq n$, then there is a subgraph $H^{\prime}$ of $H$, $\left|H^{\prime}\right|=\kappa$, in which there is an almost universal $(k-1)$-tuple.

Proof of Theorem 1, assuming Corollary 1. If $\mu=\kappa$, and $\mathbf{H}$ has an edge, then by Corollary 1 and backward induction on $k, H$ has a subgraph $H^{\prime}$ of cardinality $\kappa$ in which $\varnothing$ is almost universal. Clearly, $\varnothing$ remains almost universal in every subgraph of $H^{\prime}$ of cardinality $\kappa$. Now if $H^{\prime}$ has a nonedge, then by the dual of Corollary $1, H^{\prime}$ has a subgraph $H^{\prime \prime}$ of cardinality $\kappa$ in which $\varnothing$ is almost isolated, but this is contradictory to "almost universal," even for $\varnothing$. So $H^{\prime}$ is complete. If $\mathbf{H}$ is not complete as well, it has a nonedge $\bar{x}$, violating $H^{\prime} \cup \bar{x} \simeq H^{\prime}$.

Dually, if $\mathbf{H}$ has a nonedge, then $\mathbf{H}$ is empty. Finally, if $\mu<\kappa$, the above argument shows that every $\mu$-element subgraph of $\mathbf{H}$ is either empty or complete, whence $\mathbf{H}$ is either empty or complete.

Proof of Corollary 1, assuming the Main Lemma. If $H$ has an a.u. $k$-tuple $\bar{y}$, then so does $H(k, 2)$, because it is isomorphic to $H(k, 2) \cup \bar{y}$, and $\bar{y}$ is a.u. there.

Let $\bar{x}$ be an a.u. $k$-tuple in $H(k, 2), \bar{x}=\left\{x^{1}, \ldots, x^{k}\right\}$. We claim at least one of the $\bar{x}-\left\{x^{i}\right\}$ must extend to $\kappa$ almost universal $k$-tuples. Suppose not. Then, by removing any element outside $\bar{x}$ that could form an a.u. $\kappa$-tuple with some $\bar{x}-\left\{x^{i}\right\}$, we are removing less than $\kappa$ elements and arriving at a subgraph $H^{\prime}$ of $H(k, 2)$, with an almost universal $k$-tuple $x$ such that $v\left(\bar{x}-\left\{x^{i}\right\}\right)=1$ for all $i$. Hence $H(k, 2)$ has one as well; but this violates the main lemma, no matter how the ordering of $H(k, 2)$ is done.

Thus we can pick $\bar{x}-\left\{x^{i}\right\}$ which extends to $\kappa$ almost universal $k$-tuples. By throwing out all elements $y$ of $H$ for which $\left(\bar{x}-\left\{x^{i}\right\}\right) \cup\{y\}$ is not a.u. in $H(k, 2)$, we arrive at a subgraph $H^{\prime}$ of $H$ of cardinality $\kappa$ that has $\bar{x}-\left\{x^{i}\right\}$ for an a.u. $(k-1)$-tuple.

Proof of the Main Lemma. Fix $k$, and let $\psi: \kappa \rightarrow \bigcup\left\{[\kappa]^{j}: k-1 \leq j<n\right\}$ be a surjective function such that each element of the range has $\kappa$ elements in its preimage and such that, if $b \in[\kappa]^{j}$ and $\beta$ is the largest element of $b$, then for each cardinal $\mu$ such that $|\beta|<\mu \leq \kappa,\left|\psi^{-1}(b) \cap \mu\right|=\mu$.

We let $v_{H}$ denote valence in $H$, and let $v$ denote valence in $H(k, \lambda)$. 
Let $x_{0}, \ldots, x_{k-2}$ be arbitrary. If $v_{H}\left(x_{0}, \ldots, x_{k-2}\right)<\lambda$, let $H_{k}$ be the set of all elements $x$ such that $\left\{x_{0}, \ldots, x_{k-2}, x\right\}$ is not a.u., and let $x_{k-1} \in H_{k-1}$. Otherwise, let $H_{k}=H-\left\{x_{0}, \ldots, x_{k-2}\right\}$ and let $x_{k-1}$ be any element such that $\left\{x_{0}, \ldots, x_{k-2}, x_{k-1}\right\}$ is a.u.

Assume $\left\{x_{\beta}: \beta<\alpha\right\}$ has been chosen, along with $H_{\beta}$ whenever $k-1 \leq$ $\beta<\alpha$, so that $H_{\gamma} \supset H_{\beta}$ when $\gamma<\beta$ and $\left|H-H_{\beta}\right|<\lambda$ when $\beta<\lambda$, and of cardinality $|\beta|$ when $\beta \geq \lambda$. Let $H_{\alpha}^{\prime}$ be the set of all $y \in \bigcap_{\beta<\alpha} H_{\beta}$ such that $\bar{x} y$ is almost universal in $H$ for some $\bar{x} \subset\left\{x_{\beta}: \beta<\alpha\right\}$ satisfying $v_{H}(\bar{x})<|\alpha|^{+} \cdot \lambda$. Then $\left|H_{\alpha}^{\prime}\right|<|\alpha|^{+} \cdot \lambda$, and we let $H_{\alpha}=\bigcap_{\beta<\alpha} H_{\beta}-H_{\alpha}^{\prime}$. Of course, $H_{\alpha}$ satisfies the induction hypothesis. Let $\xi$ be the least ordinal greater than or equal to $\alpha$ for which the $j$-tuple $\bar{x}$ indexed by $\psi(\xi)$ has been defined. If $j=k-1$ and $v_{H}(\bar{x})<|\alpha|^{+} \cdot \lambda$, let $x_{\alpha}$ be any element of $H_{\alpha}$. If $v_{H}(\bar{x}) \geq|\alpha|^{+} \cdot \lambda$, let $x_{\alpha}$ be any element of $H_{\alpha}$ such that $\bar{x} x_{\alpha}$ is a.u. in $H$.

If $j \geq k$, and $\bar{x}$ is a.u. in $H$, let $x_{\alpha} \in H_{\alpha}$. If $\bar{x}$ is not a.u. in $H$, let $x_{\alpha}$ be any element of $H_{\alpha}$ such that $\bar{x} x_{\alpha}$ is not a.u. in $H$.

Let $H(k, \lambda)=\left\{x_{\alpha}: \alpha<\kappa\right\}$. Claim. If $k-1 \leq|\bar{x}| \leq n$, and $\bar{x}$ is almost universal in $H(k, \lambda)$, then $\bar{x}$ is almost universal in $H$.

Once the claim is proved, the main lemma follows thus. If $\left\{x_{\xi(1)}, \ldots, x_{\xi(k)}\right\}$ is almost universal in $H(k, \lambda)$, it is a.u. in $H$. Now, when we added $x_{\xi(k)}$ we made sure that any $(k-1)$-tuple already formed, such as $\left\{x_{\xi(1)}, \ldots, x_{\xi(k-1)}\right\}=$ $\bar{x}$, which combined with it to form an a.u. $k$-tuple in $H$, would also extend to at least $|\xi(k)|^{+} \cdot \lambda$ almost universal $k$-tuples in $H$. Then $\bar{x}$ was considered $|\xi(k)|^{+} \cdot \lambda$ times between $\xi(k)$ and $|\xi(k)|^{+} \cdot \lambda$, and each such time another element $x_{\alpha}$ was added such that $\bar{x} x_{\alpha}$ was a.u. in $H$. So the valence of $\left\{x_{\xi(1)}, \ldots, x_{\xi(k-1)}\right\}$ in $H(k, \lambda)$ is at least $|\xi(k)|^{+} \cdot \lambda$.

Proof of Claim. Argue by contrapositive and backward induction on $j$. It is obvious for $j=n$. If it has been shown for all $m>j$, and $\bar{x}$ is a $j$-tuple that is not a.u. in $H$, then $\bar{x}$ gets considered $\kappa$-many times in the induction, and each time we add a new element $x_{\alpha}$ such that $\bar{x} x_{\alpha}$ is not a.u. in $H$; hence by the backward induction hypothesis, $\bar{x} x_{\alpha}$ is not a.u. in $H(k, \lambda)$. Thus $\bar{x}$ is not a.u. in $H(k, \lambda)$.

Remarks. In the case where $\kappa$ is regular, " $\kappa$-hereditarily almost smooth" in Corollary 1 can be weakened to " $\kappa$-hereditarily $\omega$-smooth," where we call an $n$-uniform hypergraph $\omega$-smooth if it is isomorphic to each of its cofinite subgraphs. All it takes is to replace $H(k, 2)$ by $H(k, \kappa)$ in the proof of Corollary 1. Then if $\mu$ is regular we can weaken the hypothesis on Theorem 1 to " $\mu$ hereditarily $\omega$-smooth." We can do the same for singular $\mu$ if we also assume that $\left|\mathbf{I}_{\mu}(\mathbf{H})\right|<c f(\mu)$ : if we cannot have $\lambda=\mu$ in the main lemma with $\mu$ replacing $\kappa$, we can find $c f(\mu)$ nonisomorphic $H(k, \lambda)$ for some $k$, while if $\lambda=\mu$ is possible for all $k$, then Corollary 1 follows without any special hypothesis on $\left|\mathbf{I}_{\mu}(\mathbf{H})\right|$. We do not know whether the hypothesis can be dropped in general, nor, on the other hand, whether $\left|\mathbf{I}_{\mu}(\mathbf{H})\right|<c f(\mu)$ alone is enough to 
guarantee the existence of a clique or independent set of cardinality $|H|$ except, of course, in the case $c f(\mu)=\omega$ (Theorem 2).

Another problem arises naturally from the results in this section:

Problem 3. If $\mathbf{H}$ is almost smooth, must $\mathbf{H}$ have either a clique or an independent subset of cardinality $|H|$ ?

Of course, an affirmative solution to Problem 3 would lead to a very quick alternative proof of Theorem 1, as in the end of the proof assuming Corollary 1. However, on being informed of Problem 3, L. Soukup constructed a counterexample of cardinality $\omega_{1}$ in a forcing model, and Zs. Nagy constructed one assuming $\mathrm{CH}$. Both were graphs. We still do not know whether a counterexample can be constructed from ZFC alone.

Note that we cannot hope to conclude that $\mathbf{H}$ itself is empty or complete from its being almost smooth. In fact, if $\mathbf{G}=(G, E)$ is any graph, then we can replace each vertex of $\mathbf{G}$ by either a clique or an independent subset of cardinality at least $|G| \cdot \omega$, and connect two vertices by an edge iff the vertices of $\mathbf{G}$ they replace form an edge of $\mathbf{G}$. The resulting graph is almost smooth.

\section{Finite $\mathbf{I}_{\mu}(\mathbf{H})$ IMPLIES FINITE NUCLEI}

In this section, we begin our analysis of the general case where $\mathbf{I}_{\mu}(\mathbf{H})$ is finite for some infinite $\mu$, showing (Theorem 2) that $H$ must have either a clique or an independent subset $H^{\prime}$ of cardinality $|H|$ and moreover (Theorem 3) we can take $\left|H-H^{\prime}\right|<\left|\mathbf{I}_{\mu}(\mathbf{H})\right|$. Theorem 1 and the title result follow as immediate corollaries.

In Lemmas $1-3$, we will assume $\mathbf{H}=(H, E)$ is infinite and $n$-uniform, and let $\kappa=|H|$. We call $\mathbf{I}(\mathbf{H})$ minimal if it is minimal with respect to inclusion; that is, if $\mathbf{H}^{\prime}$ is an $n$-uniform hypergraph of cardinality $\kappa$ and $\mathbf{I}\left(\mathbf{H}^{\prime}\right) \subset \mathbf{I}(\mathbf{H})$, then $\mathbf{I}\left(\mathbf{H}^{\prime}\right)=\mathbf{I}(\mathbf{H})$. Of course, this is equivalent to $\mathbf{I}\left(\mathbf{H}^{\prime}\right)=\mathbf{I}(\mathbf{H})$ for all induced subgraphs $\mathbf{H}^{\prime}$ of $\mathbf{H}$ of cardinality $\kappa$, and if $\mathbf{I}(\mathbf{H})$ is finite, it is equivalent to $\left|\mathbf{I}\left(\mathbf{H}^{\prime}\right)\right|=|\mathbf{I}(\mathbf{H})|$ for all such $\mathbf{H}^{\prime}$.

Lemma 1. Suppose $\mathbf{I}(\mathbf{H})$ is minimal and finite. If $k \leq n$ and some subgraph of $\mathbf{H}$ of cardinality $\kappa$ has an almost universal $k$-tuple, then all subgraphs of cardinality $\kappa$ have at least one.

Proof. Suppose $\mathbf{H}_{1}$ has an a.u. $k$-tuple $\bar{x}_{1}$ but $\mathbf{H}_{1}^{\prime} \subset \mathbf{H}_{1}$ does not. (Of course, $k \geq 1$.) We may assume $x_{1} \cap H_{1}^{\prime}=\varnothing$ because $H_{1}^{\prime}-\bar{x}_{1}$ has a subgraph of cardinality $\kappa$ without an a.u. $k$-tuple. Then there exists $H_{2} \subset H_{1}^{\prime}$ with an a.u. $k$-tuple $\bar{x}_{2}$ as well. In general, with $H_{i}$ and $H_{i}^{\prime}$ chosen for all $i<j$, $H_{i}^{\prime} \subset H_{i} \subset H_{i-1}^{\prime}$ for all $i$, let $H_{j}$ be a subset of $H_{j-1}^{\prime}$ of cardinality $\kappa$, with an a.u. $k$-tuple $\bar{x}_{j}$. Let $H_{j}^{\prime}$ be a subset of $H_{j}$ of card $\kappa$ without an a.u. $k$-tuple, and such that $H_{j}^{\prime} \cap \bar{x}_{j}=\varnothing$.

Let $G_{m}=\bar{x}_{1} \cup \cdots \cup \bar{x}_{m} \cup H_{m}^{\prime}$. Then $G_{m}$ has the following property: by throwing out $m k$ elements, one obtains a subgraph without an a.u. $k$-tuple, 
but it is impossible to achieve this by throwing out fewer than $m$ elements. It is clear from this that there are infinitely many nonisomorphic $G_{m}$, each of cardinality $\kappa$, contradicting $\mathbf{I}(\mathbf{H})<\omega$.

We use Lemma 1 implicitly in the proof of the following lemma; the point is, $H(k, \lambda)$ could satisfy the main lemma vacuously without Lemma 1 .

Lemma 2. Suppose $\mathbf{I}(\mathbf{H})$ is minimal and finite. Let $1 \leq k \leq n$. If some subgraph of $\mathbf{H}$ of cardinality $\kappa$ has an a.u. $k$-tuple, then some subgraph of cardinality $\kappa$ has an a.u. $(k-1)$-tuple.

Proof. For regular $\kappa$, we follow the proof of Corollary 1, except that we use $\mathbf{H}(k, \kappa)$ instead of $\mathbf{H}(k, 2)$ and dispense with all but the first sentence of the second paragraph. For singular $\kappa$, let $\bar{x}$ be an almost universal $k$-tuple of $\mathbf{H}^{\prime} \subset \mathbf{H}$. If for some $x^{i} \in \bar{x}, \bar{x}-\left\{x^{i}\right\}$ extends to $\kappa$ almost universal $k$-tuples of $\mathbf{H}^{\prime}$, the proof of Corollary 1 again goes through and we get $\mathbf{H}^{\prime \prime}$ with an a.u. $(k-1)$-tuple. So suppose every a.u. $k$-tuple $\bar{x}$ of every $\mathbf{H}^{\prime} \subset \mathbf{H},\left|H^{\prime}\right|=\kappa$ fails to have such an element $x^{i}$. Then for each $\lambda<\kappa$, the minimum of all the $\max \left\{v_{\mathbf{H}(k, \lambda)}\left(\bar{x}-\left\{x^{i}\right\}\right): 1 \leq i \leq k\right\}$ as $\bar{x}$ ranges over the a.u. $k$-tuples of $\mathbf{H}(k, \lambda)$ is at least $\lambda$, but less than $\kappa$, and so there are infinitely many nonisomorphic $\mathbf{H}(k, \lambda)$, contradicting the hypothesis on $\mathbf{H}$.

Lemma 3. Suppose $\mathbf{I}(\mathbf{H})$ is minimal and finite. If $\mathbf{H}$ has an edge, $\varnothing$ is almost universal in $\mathbf{H}$.

Proof. Repeated applications of Lemma 2 give a subgraph $\mathbf{H}^{\prime}$ of $\mathbf{H}$ with an almost universal 0-tuple, namely $\varnothing$. Now $\mathbf{H}^{\prime}$ has a subgraph isomorphic to $\mathbf{H}$ so $\varnothing$ is almost universal in $\mathbf{H}$.

Theorem 2. If $\mathbf{H}=(H, E)$ is an infinite n-uniform hypergraph with $\mathbf{I}(\mathbf{H})$ finite, then $\mathbf{H}$ has either a clique or an independent subset of cardinality $|H|$.

Proof. Suppose not, and let $\mathbf{H}$ be a counterexample with $|\mathbf{I}(\mathbf{H})|$ minimal. Then $\mathbf{I}(\mathbf{H})$ itself is minimal. Since $\mathbf{H}$ has an edge, $\varnothing$ is almost universal; dually, since $\mathbf{H}$ has a nonedge, $\varnothing$ is almost isolated; but these are contradictory conclusions.

Corollary 2. If $\mathbf{I}(\mathbf{H})$ is minimal and finite, then $|\mathbf{I}(\mathbf{H})|=1$ and $\mathbf{H}$ is either empty or complete.

The subset promised by Theorem 2 is "almost all" of $H$, according to Theorem 3. To prove it, we introduce some more concepts.

Definition 3. Let $\mathbf{H}=(H, E)$ be a hypergraph. A subset $K$ of $H$ is a cover of $\mathbf{H}$ if $\mathbf{H}[(H-K)$ is empty. A cover $K$ is called a nucleus if no subset of $H$ of strictly smaller cardinality is a cover and minimal if no proper subset is a cover. We let nuc $(\mathbf{H})$ denote the cardinality of a nucleus of $\mathbf{H}$.

Note that $K$ is a cover iff $K$ meets each $e \in E$. A routine Zorn's lemma argument shows that every cover has a minimal subcover. 
Lemma 4. If an n-uniform hypergraph has a finite cover, it has only finitely many nuclei.

Proof. Let $m$ be the cardinality of any nucleus of $\mathbf{H}$. There cannot be enough nuclei to form a $\Delta$-system of cardinality $n+1$, otherwise each edge must meet the root of the system, making the root a cover of cardinality $<m$, contradicting the definition of a nucleus. But every collection of infinitely many sets of cardinality $m$ contains an infinite $\Delta$-system.

[Aside. By way of contrast, the disjoint union of $\lambda$ two-element cliques is a graph with $2^{\lambda}$ minimal nuclei.]

Proof of Theorem 3. By Theorem 2, we may assume without loss of generality that $H$ has an independent subset $H_{0}$ of cardinality $\mu$. Of course, $\varnothing$ is the unique nucleus of $H_{0}$. If $\mathbf{H}$ has any edges, adjoining the elements of an edge to $H_{0}$ one at a time will raise the nucleus size of the resulting subgraph. At the step when it first becomes nonzero, it equals 1 , because the last element is a singleton nucleus; call the resulting subgraph $\mathbf{H}_{1}$.

In general, suppose we have added finitely many elements to $H_{0}$, arriving at a subgraph $\mathbf{H}_{k}$ whose nuclei are of cardinality $k$ (finite). There are only finitely many, by Lemma 4 , and we can destroy each by adding an edge of $\mathbf{H}$ disjoint from it, unless there is no such edge, in which case the $k$-tuple will be a nucleus of $\mathbf{H}$. The destruction can be completed by adding finitely many elements, and if we add them one at a time, then the element $x$ that destroys the "last" nucleus $K_{k}$ of size $k$ gives a nucleus $K_{k} \cup\{x\}$ of the new subgraph $\mathbf{H}_{k+1}$ of size $k+1$.

But this process must cease before we arrive at $k=\mathbf{I}_{\mu}(\mathbf{H})$, because $\mathbf{H}_{i}$ cannot be isomorphic to $\mathbf{H}_{j}$ if $i \neq j: \operatorname{nuc}\left(\mathbf{H}_{i}\right)$ is an isomorphism invariant. Hence $\mathbf{H}$ has a nucleus of cardinality at most $\mathbf{I}_{\mu}(\mathbf{H})-1$.

Corollary 2 naturally gives rise to a number of questions.

Problem 4. Does there exist a hypergraph of regular cardinality with infinite minimal $\mathbf{I}(\mathbf{H})$ (equivalently, minimal $\mathbf{I}(\mathbf{H})$ and $|\mathbf{I}(\mathbf{H})|>1$ ) ? countably infinite minimal $\mathbf{I}(\mathbf{H})$ ?

Of course, such examples would be uncountable by Ramsey's theorem. For each singular cardinal $\kappa$, there is an elementary example of minimal $\mathbf{I}(\mathbf{H})$ with $|\mathbf{I}(\mathbf{H})|=\chi(\kappa)^{c f(\kappa)}$ : the direct sum of $c f(\kappa)$ cliques of cardinality less than $\kappa$, the supremum of whose cardinalities is $\kappa$. However, at this point we do not even know whether it is consistent that these examples, and their duals, together with empty and complete hypergraphs, exhaust the list of $\mathbf{H}$ with minimal $\mathbf{I}(\mathbf{H})$. In [1], Baumgartner constructs a Souslin tree, assuming $\uparrow^{+}$, whose comparability graph has minimal $I(H)$. But $I(H) \geq c$ for each Souslin tree; see $\S 5$.

Problem 5. Does there exist a hypergraph of singular cardinality $\kappa$ with minimal $\mathbf{I}(\mathbf{H})$ of cardinality greater than $\chi(\kappa)^{c f(\kappa)}$ ? 
At the opposite extreme, the following problem also seems to be open:

Problem 6. Is there an infinite hypergraph $\mathbf{H}$ such that no subgraph $\mathbf{H}^{\prime}$ of cardinality $|H|$ has minimal $\mathbf{I}\left(\mathbf{H}^{\prime}\right)$ ?

\section{THE FUNDAMENTAL CORRESPONDENCE}

For all its strength, Theorem 3 still leaves a great deal unsaid about hypergraphs with finite $\mathbf{I}(\mathbf{H})$. For instance, it does not seem to easily imply that they are $\omega$-polarized:

Definition 4. An infinite $n$-uniform hypergraph $\mathbf{H}=(H, E)$ is polarized if every $k$-tuple, $0 \leq k \leq n$, of $\mathbf{H}$ is either almost isolated or almost universal. If $\lambda$ is a cardinal number, then $\mathbf{H}$ is $\lambda$-polarized if it is polarized and $d(\bar{x})<\lambda$ for all $k$-tuples $\bar{x}, 0 \leq k<n$.

It is fairly easy to see that this is true for graphs $(n=2)$ and that, conversely, an $\omega$-polarized graph with a finite nucleus has only finitely many nonisomorphic induced subgraphs of any fixed cardinality. This converse does not hold for any $n>2$, however. To give a characterization valid for all $n$, we introduce in this section some machinery that will be used to prove Theorems 4,5 , and 6 and the reduction theorems mentioned in the introduction. These reduction theorems are part of a more general functorial correspondence between $\lambda$-polarized $n$ uniform hypergraphs $\mathbf{H}=(H, E)$ and $\lambda$-bounded hypergraphs, $\mathbf{F}=(H, \widetilde{E})$, of order at most $n$ with the same underlying set $H$.

Definition 5. Let $\mathbf{F}=(H, \widetilde{E})$ be a (not necessarily uniform) hypergraph. Let $\bar{x}$ be a $k$-tuple in $H$. The degree of $\bar{x}$ is $\delta(\bar{x})=|\{y: \bar{x} y \in \widetilde{E}\}| . F$ is $\lambda$-bounded if $\delta(\bar{x})<\lambda$ for every tuple $\bar{x}$ in $H$. If $H$ is infinite, we say $\mathbf{F}$ is bounded if it is $|H|$-bounded.

In the following section, we will show that, under this correspondence, if $\mathbf{I}_{\mu}(\mathbf{H})=N$ for some positive integer $N$ and infinite $\mu$, then $\mathbf{H}$ is $N$-polarized and corresponds to a hypergraph with only finitely many edges.

To help understand the correspondence, we first consider the case of infinite graphs $\mathbf{G}$ with finite $\mathbf{I}(\mathbf{G})$. If $\varnothing$ is a.i., there can be only finitely many a.u. singletons, since these are part of any nucleus. Also, any vertex with infinitely many edges emanating from it is part of every nucleus, and if it is not a.u. of finite deficiency, it is easy to come up with infinitely many pairwise nonisomorphic subgraphs. It is now easy to see that there are only finitely many edges in all which do not contain an a.u. singleton and only finitely many pairs which are not edges and do contain an a.u. singleton. By listing these finitely many "exceptional" pairs and the finitely many "exceptional" a.u. singletons, we can now reconstruct the entire graph, given its underlying set.

If $\varnothing$ is a.u., the criteria of whether a singleton and a pair are "exceptional" are the dual of the above. In general, the idea is to let the corresponding hypergraph have as edges the "most exceptional" $k$-tuples, using these as a catalogue from 
which to reconstruct the original hypergraph. If $n>2$, we need an index of how "exceptional" a $k$-tuple is, if we are to keep the catalogue finite, even in the case of finite $\mathbf{I}(\mathbf{H})$. This consideration and ease of recovery of $\mathbf{H}$ from the catalogue led the authors naturally (at least in the case of polarized $\mathbf{H}$ ) to the following "exceptionality index."

Definition 6. If $\mathbf{H}=(H, E)$ is $n$-uniform, the large index $I_{\mathbf{H}}(\bar{x})=I(\bar{x})$ of a $k$-tuple $\bar{x}$ in $\mathbf{H}$ is defined by induction on $|\bar{x}|$ as follows:

(0) $J(\varnothing)=0$,

(1) $J(\bar{x})=\max \{I(\bar{y}): \bar{y} \subset \bar{z}$ and $y \neq z\}$ if $|\bar{x}|>0$,

(2)

$$
I(\bar{x})= \begin{cases}J(\bar{x}) & \text { if either } J(\bar{x}) \text { is odd and } \bar{x} \text { is a.u. } \\ J(\bar{x})+1 & \text { or } J(\bar{x}) \text { is even and } \bar{x} \text { is not a.u. }\end{cases}
$$

Clearly $I(\bar{x})$ is odd iff $\bar{x}$ is almost universal, and $I(\bar{x}) \neq J(\bar{x})$ iff $I(\bar{x})=$ $J(\bar{x})+1$.

For the remainder of this section, $\mathbf{H}$ will denote a polarized $n$-uniform hypergraph $(H, E)$.

Definition 7. The signature $\mathbf{S}(\mathbf{H})=\left(H, \widetilde{E}_{\mathbf{H}}\right)$ of $\mathbf{H}$ is defined by $\widetilde{E}_{\mathbf{H}}=\{\bar{x} \subset$ $H: I(\bar{x})=J(\bar{x})+1\}$.

Lemma 5. If $\mathbf{H}$ is $\lambda$-polarized then $\mathbf{S}(\mathbf{H})$ is $2^{n-1}(\lambda-1)+1$-bounded.

Proof. First we prove: if $J(\bar{x} y)<I(\bar{x} y)$ then $I(\bar{w})=J(\bar{w} y)<I(\bar{w} y)$ for some $\bar{w} \subset \bar{x}$. Argue by induction on $|\bar{x}|$. It is clearly true if $\bar{x}=\varnothing$. If $I(\bar{x})=J(\bar{x} y)$, let $\bar{w}=\bar{x}$. Now suppose $I(\bar{x})<J(\bar{x} y)$. Let $\bar{w}$ be a minimal (proper) subset of $\bar{x}$ such that $J(\bar{x} y)=I(\bar{w} y)$. Then $J(\bar{w} y)<I(\bar{w} y)$ since $I(\bar{w}) \leq I(\bar{x})<J(\bar{x} y)=I(\bar{w} y)$ and the choice of $\bar{w}$ guarantees that $I(\bar{v} y)<$ $I(\bar{w} y)$ for all proper subsets $\bar{v}$ of $\bar{w}$. Now by the induction hypothesis, $I(\bar{u})=$ $J(\bar{u} y)<I(\bar{u} y)$ for some $\bar{u} \subset \bar{w} \subset \bar{x}$ and we are done.

Fix $\bar{x}$. If $\bar{x} y \in \widetilde{E}_{\mathbf{H}}$ then $J(\bar{x} y)<I(\bar{x} y)$. Thus there exists $\bar{w} \subset \bar{x}$ such that $I(\bar{w})=J(\bar{w} y)<I(\bar{w} y)$. Since $\mathbf{H}$ is $\lambda$-polarized there are fewer than $\lambda$ such $y$ for each $\bar{w} \subset \bar{x}$. Thus $\delta(\bar{x})<2^{n-1}(\lambda-1)+1$.

Now we define an index which will enable us to recapture any polarized $\mathbf{H}$ from its signature and also to establish that any bounded infinite hypergraph with edges of cardinality at most $n$ can be the signature of a polarized $n$-uniform hypergraph. However, this index also behaves well for arbitrary hypergraphs, so at first we make no assumptions about $\mathbf{F}=(H, \widetilde{E})$ despite the suggestive $\widetilde{E}$ notation. We do not even assume our $k$-tuples are subsets of $H$.

Definition 8. The small index $i_{\mathbf{F}}(\bar{x})=i(\bar{x})$ of a finite set $\bar{x}$ is defined by induction on $\bar{x}$ as follows:

(0) $j(\varnothing)=0$,

(1) $j(\bar{x})=\max \{i(\bar{y}): \bar{y} \subset \bar{x}$ and $y \neq x\}$ if $|\bar{x}|>0$, 
(2)

$$
i(\bar{x})= \begin{cases}j(\bar{x}) & \text { if } \bar{x} \notin \widetilde{E} \\ j(\bar{x})+1 & \text { otherwise. }\end{cases}
$$

Note that $i(\bar{x}) \neq j(\bar{x})$ iff $i(\bar{x})=j(\bar{x})+1$ iff $\bar{x} \in \widetilde{E}$.

Definition 9. Let $\mathbf{F}=(H, \widetilde{E})$ be a hypergraph and let $G$ be a set. The parity transform $\mathbf{T}(G, \mathbf{F})$ is the hypergraph whose vertex set is $G$ and whose set of edges is $\widetilde{\mathbf{T}}(G, \mathbf{F})=\left\{\bar{x} \subset G:|\bar{x}|\right.$ is finite and $i_{\widetilde{E}}(\bar{x})$ is odd $\}$. The $n$-uniform parity transform $\mathbf{T}_{n}(G, \mathbf{F})$ is $\left(G, \widetilde{\mathbf{T}}(G, \mathbf{F}) \cap[G]^{n}\right)=\left(G, \widetilde{\mathbf{T}}_{n}(G, \mathbf{F})\right)$. In case $G=H$ we write $\mathbf{T}(\mathbf{F}), \widetilde{\mathbf{T}}(\mathbf{F}), \mathbf{T}_{n}(\mathbf{F})$, and $\widetilde{\mathbf{T}}_{n}(\mathbf{F})$. If $n$ is understood we write $\widetilde{\mathbf{T}}_{n}(G, \mathbf{F})=\widetilde{E}_{G}^{\#}$ and $\widetilde{\mathbf{T}}_{n}(\mathbf{F})=\widetilde{E}^{\#}$.

It can be shown that $\mathbf{T}$ is an automorphism of the category of all hypergraphs and their embeddings and that it has infinite order under composition. However, in this paper we are concerned with $\mathbf{T}_{n}$ rather than $\mathbf{T}$.

Note that if $G \subset H$, then $\mathbf{T}(G, \mathbf{F})=\mathbf{T}\left(\mathbf{F}\lceil G)\right.$ : on subsets of $G, i_{\mathbf{F}}$ and $i_{\mathrm{F}\lceil G}$ agree. More generally, $\mathrm{T}(G, \mathbf{F})=\mathrm{T}(G, \mathbf{F}\lceil G \cap H)$ by the same reasoning.

Lemma 6. If $\mathbf{F}$ is $\lambda$-bounded and $|G| \geq 2^{n}(\lambda-1)+n$, then $\mathbf{T}_{n}(G, \mathbf{F})$ is $2^{n-1}(\lambda-1)+1$-polarized. Moreover $\bar{x}$ is almost universal in $\mathbf{T}_{n}(G, \mathbf{F})$ iff $i_{\mathbf{F}}(\bar{x})$ is odd iff $\bar{x}$ is not almost isolated in $\mathbf{T}_{n}(G, \mathbf{F})$.

Proof. First we show that for any $k$-tuple $\bar{x}, 0 \leq k<n$ in $G, i(\bar{x} y)=i(\bar{x})$ for all but fewer than $2^{k}(\lambda-1)+1$ elements $y$ of $G$. Suppose that $i(\bar{x} y)>$ $i(\bar{x})$. Let $\bar{w}$ be a minimum subset of $\bar{x}$ such that $i(\bar{w} y)=i(\bar{x} y)$. Then $j(\bar{w} y)<i(\bar{w} y)$. Thus $\bar{w} y \in \widetilde{E}$. Since $\mathbf{F}$ is $\lambda$-bounded, there are fewer than $\lambda$ of these $y$ for each subset $\bar{w}$, so we are done.

Next we show that $\bar{x}$ is almost universal in $\mathbf{T}_{n}(G, \mathbf{F})=\left(G, \widetilde{E}_{G}^{\#}\right)$ if $i(\bar{x})$ is odd and almost isolated if $i(\bar{x})$ is even. We argue by reverse induction on $|\bar{x}|=k$. If $k=n$ then $\bar{x}$ is almost universal in $\left(G, \widetilde{E}_{G}^{\#}\right)$ iff $\bar{x} \in \widetilde{E}_{G}^{\#}$ iff $i(\bar{x})$ is odd. Now assume the claim has been proved for $k+1$. If $i(\bar{x})$ is odd then $i(\bar{x} y)$ is odd for all but fewer than $(\lambda-1) 2^{n-1}+1$ elements of $G$. Thus by the inductive hypothesis and the size of $G, \bar{x}$ is almost universal in $\left(G, \widetilde{E}_{G}^{*}\right)$. A similar argument holds if $i(\bar{x})$ is even.

Lemma 7. $I_{\mathbf{H}}(\bar{x})=i_{\mathbf{S}(\mathbf{H})}(\bar{x})$ for all $\bar{x} \in H, 0 \leq|\bar{x}| \leq n$.

Proof. We show by induction on $|\bar{x}|$, suppressing the respective subscripts, that $J(\bar{x})=j(\bar{x})$ and $I(\bar{x})=i(\bar{x})$. Clearly $J(\varnothing)=0=j(\varnothing)$. Now assume $J(\bar{x})=j(\bar{x})$ for all $k$-tuples $\bar{x}$ in $H . \quad I(\bar{x})=J(\bar{x})+1$ iff $\bar{x} \in \widetilde{E}_{\mathbf{H}}$ iff $i(\bar{x})=j(\bar{x})+1$. Thus $I(\bar{x})=i(\bar{x})$, and so $J(\bar{y})=j(\bar{y})$ for all $(k+1)$-tuples $\bar{y}$.

Lemma 8. If $\mathbf{F}$ is $\lambda$-bounded and $|G| \geq 2^{n}(\lambda-1)+n$, then $i_{\mathbf{F}}(\bar{x})=I_{\mathbf{D}}(\bar{x})$, $\mathbf{D}=T_{n}(G, \mathbf{F})$ for all $\bar{x} \subset G, 0 \leq|\bar{x}| \leq n$.

Proof. The size of $G$ assures that $\mathbf{T}_{n}(G, \mathbf{F})$ is polarized. We shall show by induction on $|\bar{x}|$ that $j(\bar{x})=J(\bar{x})$ and $I(\bar{x})=i(\bar{x})$. Clearly $j(\varnothing)=J(\varnothing)$. 
Now assume that $j(\bar{x})=J(\bar{x})$ for all $k$-tuples $\bar{x}$. Then $i(\bar{x})=j(\bar{x})+1$ iff either $i(\bar{x})$ is even and $j(\bar{x})$ is odd or $i(\bar{x})$ is odd and $j(\bar{x})$ is even iff either $\bar{x}$ is a.i. and $J(\bar{x})$ is odd or $\bar{x}$ is a.u. and $J(\bar{x})$ is even iff $I(\bar{x})=J(\bar{x})+1$. Thus $i(\bar{x})=I(\bar{x})$ and so $j(\bar{y})=J(\bar{y})$ for all $(k+1)$-tuples $\bar{y}$.

Definition 10. Let $\lambda$ be an infinite cardinal. Let $\mathbf{P}_{\lambda}$ be the class of all $\lambda$ polarized $n$-uniform hypergraphs of cardinality at least $\lambda$ and $\mathbf{B}_{\lambda}$ the class of all $\lambda$-bounded hypergraphs of cardinality at least $\lambda$ and order at most $n$. Let $\mathbf{S}: \mathbf{P}_{\lambda} \rightarrow \mathbf{B}_{\lambda}$ be the class function taking each $\mathbf{H}$ to $\mathbf{S}(\mathbf{H})$, and $\mathbf{T}_{n}: \mathbf{B}_{\lambda} \rightarrow \mathbf{P}_{\lambda}$ be the one taking $\mathbf{F}$ to $\mathbf{T}_{n}(\mathbf{F})$.

Theorem 7. Let notation be as above. Let $\mathbf{H}=(H, E) \in \mathbf{P}_{\lambda}, \mathbf{F}=(H, \widetilde{E}) \in B_{\lambda}$.

(1) $\mathbf{T}_{n} \circ \mathbf{S}=\mathrm{id}=\mathbf{S} \circ \mathbf{T}_{n}$.

(2) Let $H^{\prime} \subset H$.

(a) $\mathbf{S}\left(\mathbf{H}\left\lceil\mathbf{H}^{\prime}\right)=\mathbf{S}(\mathbf{H})\left\lceil H^{\prime}\right.\right.$ if $\left|H^{\prime}\right| \geq \lambda$.

(b) $\mathbf{T}_{n}\left(\mathbf{F}\left\lceil H^{\prime}\right)=\mathbf{T}_{n}(\mathbf{F})\left\lceil H^{\prime}\right.\right.$.

(3) (a) $\mathbf{H} \simeq \mathbf{H}^{\prime}$ iff $\mathbf{S}(\mathbf{H}) \simeq \mathbf{S}\left(\mathbf{H}^{\prime}\right)$.

(b) $\mathbf{F} \simeq \mathbf{F}^{\prime}$ iff $\mathbf{T}_{n}(\mathbf{F}) \simeq \mathbf{T}_{n}\left(\mathbf{F}^{\prime}\right)$.

(4) Let $H_{0}, H_{1}$ be subsets of $H,\left|H_{i}\right| \geq \lambda$.

(a) $\mathbf{H}\left\lceil H_{0} \simeq \mathbf{H}\left\lceil H_{1}\right.\right.$ iff $\mathbf{S}(\mathbf{H})\left\lceil H_{0} \simeq \mathbf{S}(\mathbf{H})\left\lceil H_{1}\right.\right.$.

(b) $\mathbf{F}\left\lceil H_{0} \simeq \mathbf{F}\left\lceil H_{1}\right.\right.$ iff $\mathbf{T}_{n}(\mathbf{F})\left\lceil\mathbf{H}_{0} \simeq \mathbf{T}_{n}(\mathbf{F})\left\lceil H_{1}\right.\right.$.

(5) If $\lambda \leq \mu \leq|H|$, then

(a) $\left|\mathbf{I}_{\mu}(\mathbf{H})\right|=\left|\mathbf{I}_{\mu}(\mathbf{S}(\mathbf{H}))\right|$ and

(b) $\left|\mathbf{I}_{\mu}(\mathbf{F})\right|=\left|\mathbf{I}_{\mu}\left(\mathbf{T}_{n}(\mathbf{F})\right)\right|$.

Proof. (1) Use Lemmas 7 and 8.

(2a) Note that $\left|H^{\prime}\right|$ was chosen large enough so that $I_{\mathbf{H}}=I_{\mathbf{H}^{\prime}}$ on $H^{\prime}$. [As usual, we let $\mathbf{H}^{\prime}=\mathbf{H}\left[H^{\prime}\right.$.] Thus $\bar{x} \in \widetilde{E}_{\mathbf{H}^{\prime}}$ iff $J_{\mathbf{H}^{\prime}} \neq I_{\mathbf{H}^{\prime}}$ iff $J_{\mathbf{H}} \neq I_{\mathbf{H}}$, iff $\bar{x} \in \widetilde{E}_{\mathbf{H}}\left\lceil H^{\prime}\right.$.

(2b) Here the size of $H^{\prime}$ does not matter. By the note preceding Lemma 6, $\mathbf{T}_{n}\left(\mathbf{F}\left\lceil\mathbf{H}^{\prime}\right)=\mathbf{T}_{n}\left(H^{\prime}, \mathbf{F}\right)\right.$ and the small indices agree on finite subsets $\bar{x}$ of $H^{\prime}$. Thus $\bar{x} \in \widetilde{E}_{\mathbf{H}^{\prime}}^{\#}$ iff $|\bar{x}|=n$ and $i(\bar{x})$ is odd iff $\bar{x} \in \widetilde{E}_{\mathbf{H}}^{\#}$.

(3) The "if" directions are obvious. For the "only if" directions, use (1).

(4) Use (2) and (3). The size of $H_{0}$ and $H_{1}$ does matter in the "only if" direction of (a) and the "if" direction of (b).

(5) Use (4).

Theorem 8. If $\mathbf{S}(\mathbf{H})$ has finitely many edges, then $\mathbf{I}_{\mu}(\mathbf{H})$ is finite for all cardinals $\mu$. Moreover, if $\mu$ and $\nu$ are infinite cardinals and $\mu, \nu \leq|H|$, then $\left|I_{\mu}(\mathbf{H})\right|=$ $\left|I_{\nu}(\mathbf{H})\right|$.

Proof. Since $\mathbf{S}(\mathbf{H})$ is finite, $\mathbf{S}(\mathbf{H})$ is $\omega$-bounded. It follows from the definition of $\mathbf{S}(\mathbf{H})$ that $\mathbf{H}$ is $\omega$-polarized. Thus the first statement is immediate from 5(a). To show the second, we first establish some notation. If $\mathbf{H}^{\prime}$ is an induced subgraph of $\mathbf{H}$, we write $F_{\mathbf{H}^{\prime}}$ for $\bigcup \widetilde{E}_{\mathbf{H}^{\prime}}$, and we let $\mathbf{F}\left(\mathbf{H}^{\prime}\right)$ be the finite hypergraph $\left(F_{\mathbf{H}^{\prime}}, \widetilde{E}_{\mathbf{H}^{\prime}}\right)$. Now if $\left|H^{0}\right|=\left|H^{1}\right| \geq \omega$ then $\mathbf{H}^{0} \simeq \mathbf{H}^{1}$ iff 
$F\left(\mathbf{H}^{0}\right) \simeq F\left(\mathbf{H}^{1}\right)$ : "only if" is clear while "if" follows from the fact that $I_{\mathbf{H}^{i}}$ and $i_{\mathbf{F}\left(\mathbf{H}^{i}\right)}$ agree on $H^{i}$, so that $\mathbf{H}^{i}=\mathbf{T}_{n}\left(H^{i}, F\left(\mathbf{H}^{i}\right)\right)$, and the isomorphism type of $\mathbf{T}_{n}\left(H^{i}, \mathbf{F}\left(\mathbf{H}^{i}\right)\right)$ depends only upon that of $\mathbf{F}\left(\mathbf{H}^{i}\right)$ and the cardinality of $H^{i}-F_{\mathbf{H}^{0}}$, and these agree by finiteness of $F_{\mathbf{H}^{i}}$. Hence there is a natural bijection between $\mathbf{I}_{\mu}(\mathbf{H})$ for each infinite $\mu$ and the isomorphism classes of induced finite subgraphs $\left(F_{\mathrm{A}}, \widetilde{E}_{\mathbf{A}}\right)$ for $A \subset F_{\mathbf{H}}$.

In the following section, we will see that the converse of the first sentence of Theorem 8 holds. More strongly, we show:

Lemma 9. If $I_{\mu}(\mathbf{H})$ is finite for some infinite $\mu \leq|H|$, then $\mathbf{S}(\mathbf{H})$ has finitely many edges; moreover, $d(\bar{x})<\left|\mathbf{I}_{\mu}(\mathbf{H})\right|$ for every $k$-tuple $\bar{x}, 0 \leq k<n$.

Theorems 4, 5, and 6 follow immediately from Theorem 8 and Lemma 9; in particular, we can recapture $\mathbf{H}$ from any $\mu$-element subgraph $\mathbf{H}^{0}$ that contains $F_{\mathbf{H}^{\prime}}$ by simply taking $\mathrm{T}_{n}\left(H, S\left(\mathbf{H}^{0}\right)\right)$. Such subgraphs are distinguished by the fact that they have the greatest $F_{\mathbf{H}}^{0}$ of all subgraphs of cardinality $\mu$.

The conclusion in the second sentence of Theorem 8 can be extended downward to finite $\mu$ and $\nu$; just how far varies from one $\mathbf{H}$ to another and will be dealt with in a future paper. Of course, some $\mu$ and $\nu$ are easily seen to be "safe" by various results of this section. For instance, if $\lambda$ is a (finite) cardinal and $\mathbf{S}(\mathbf{H})$ is $\lambda$-bounded, $\mu, \nu \geq 2^{n}(\lambda-1)+n$ is "safe" by Lemma 6 .

We close this section with some categorical remarks that will not be needed later.

From Theorem 7 and the following theorem, it is clear that $\mathbf{S}$ and $\mathbf{T}_{n}$ can be regarded as functors between the category $\left(\mathbf{P}_{\lambda}\right.$, embeddings $)$ and $\left(\mathbf{B}_{\lambda}\right.$, embeddings), and hence the two categories are isomorphic. Conversely, the fact that $\mathbf{S}$ and $\mathbf{T}_{n}$ are inverse isomorphisms of these categories implies most of Theorems 7 and 9; we leave it to the interested reader to verify this and to try to formulate a category-theoretic statement implying all parts.

Theorem 9. Let $\mathbf{H}, \mathbf{H}^{\prime} \in \mathbf{P}_{\lambda}$ and let $\mathbf{F}, \mathbf{F}^{\prime}$ be hypergraphs.

(1) If $f: H \rightarrow H^{\prime}$ is a 1-1 function, then $f$ is an embedding of $\mathbf{H}$ into $\mathbf{H}^{\prime}$ iff $f$ is an embedding of $\mathbf{S}(\mathbf{H})$ into $\mathbf{S}\left(\mathbf{H}^{\prime}\right)$.

(2) If $f$ is an embedding of $\mathbf{F}$ into $\mathbf{F}^{\prime}$, then $f$ is an embedding of $\mathbf{T}_{n}(\mathbf{F})$ into $\mathbf{T}_{n}\left(\mathbf{F}^{\prime}\right)$ for all $n \in \omega$.

Proof. By Theorem 7 and the fact that embeddings preserve $i$, and also $I$ if both the domain and codomain are $\lambda$-polarized.

The converse of (2) is also true; one does need $f$ to be an embedding for all finite $n \leq|F|$, however. This is what is behind our earlier statement that $\mathbf{T}$ is 
an automorphism of the category of all hypergraphs and their embeddings; but we leave the proof for a future paper.

\section{THE REDUCTION COMPLETED}

In this section, we establish Lemma 9. The argument we give is a simplification of our original proof suggested by $\mathbf{M}$. Pouzet. As usual, we let $\mathbf{H}=(H, E)$ be an $n$-uniform hypergraph.

Definition 11. If $y \in H$, then $\mathbf{H}(y)=\mathbf{H}\lceil(H-\{y\})$. If $F$ is a subset of $H$ of (finite) cardinality $f$ less than $n$, then $\mathbf{H} / F$ is the $(n-f)$-uniform hypergraph defined on $H-F$ by: $e$ is an edge of $\mathbf{H} / F$ iff $e \cup F$ is an edge of $\mathbf{H}$.

We omit the routine proof of

Lemma 10. (a) If $y \in \mathbf{H}, \mathbf{H}(y)$ is polarized, and $\bar{x}$ is a $k$-tuple in $\mathbf{H}(y)$, then $d_{\mathbf{H}}(\bar{x}) \leq d_{\mathbf{H}(y)}(\bar{x})+1$; furthermore, if $\mathbf{H}$ is also polarized then $\bar{x} \in \widetilde{E}_{\mathbf{H}(y)}$ iff $\bar{x} \in \widetilde{E}_{\mathbf{H}}$.

(b) If $F$ is a subset of $H$ of cardinality $f<n, \mathbf{H} / F$ is polarized, and $\bar{x}$ is a $k$-tuple in $H-F$ with $k \leq n-f$, then $d_{\mathbf{H}}(\bar{x} \cup F)=d_{\mathbf{H} / F}(\bar{x})$. Furthermore, if $\mathbf{H}$ is polarized and $\bar{x} \neq \varnothing$, then $\bar{x} \in \widetilde{E}_{\mathbf{H} / F}$ iff $\bar{x} \cup F \in \widetilde{E}_{\mathbf{H}}$; in fact, $I_{\mathbf{H} / F}(\bar{x})+$ $I_{\mathbf{H}}(F)=I_{\mathbf{H}}(\bar{x} \cup F)$. Also, $\varnothing$ is almost universal in $\mathbf{H} / F$ iff $F$ is almost universal in $\mathbf{H}$.

Proof of Lemma 9. By Theorem 3, either $\mathbf{H}$ or its dual has a finite nucleus $K$; without loss of generality, assume it is $\mathbf{H}$. We argue by induction on $N=\left|\mathbf{I}_{\mu}(\mathbf{H})\right|$. If $N=1$ then $\mathbf{H}$ is empty by Theorem 1 . Thus $d(\bar{x})=0$ and $\widetilde{E}_{\mathbf{H}}=\varnothing$. Suppose the lemma holds for $N<M$ and set $N=M$. Now we argue by induction on $n$. If $n=1$ the lemma is obvious, so we assume the lemma holds for $N=M$ and $n<m$. Let $\delta \mathbf{H}$ be the set of all isomorphism classes of finite induced subgraphs of $\mathbf{H}$, and let $Y_{\mathbf{H}}$ denote $\{x \in H: \delta \mathbf{H} \neq \delta \mathbf{H}(x)\}$. Note that $Y_{\mathbf{H}}$ is nonempty since every nucleus is a subset of $Y_{\mathbf{H}}$.

Claim. If $Y_{\mathbf{H}} \subset P \subset H$, where $|P|=\mu$, then $Y_{\mathbf{P}}=Y_{\mathbf{H}}$ and $\delta \mathbf{P}=\delta \mathbf{H}$.

Proof of Claim. We show that (1) if $Y_{\mathbf{P}} \neq Y_{\mathbf{H}}$ then $\delta \mathbf{P} \neq \delta \mathbf{H}$ and (2) if $\delta \mathbf{P} \neq \delta \mathbf{H}$ then there exists $\mathbf{P}^{\prime}$ such that $P \subset P^{\prime} \subset H, P^{\prime}-P$ is finite, $\delta \mathbf{P} \neq \delta \mathbf{P}^{\prime}$, and $Y_{\mathbf{P}^{\prime}} \neq Y_{\mathbf{H}}$. So, if either conclusion of the claim is violated, we can build a chain $P_{0} \subset P_{1} \subset \cdots \subset H$, where $P_{0}=P$ and $P_{i+1}=P_{i}^{\prime}$, such that $\left|P_{i}\right|=\mu$ and $\delta \mathbf{P}_{i} \neq \delta \mathbf{P}_{j}$ for all $i<j<\omega$, which contradicts the finiteness of $\mathbf{I}_{\mu}(\mathbf{H})$.

For (1), first suppose that $x \in Y_{\mathbf{H}}-Y_{\mathbf{P}}$. Then there exists an isomorphism type $\tau \in \delta \mathbf{H}-\delta \mathbf{H}(x)$. Clearly, $\tau \notin \delta \mathbf{P}(x)$. Since $x \notin Y_{\mathbf{P}^{\prime}}, \tau \notin \delta \mathbf{P}$ and thus $\delta \mathbf{H} \neq \delta \mathbf{P}$. Second, suppose that $x \in Y_{\mathbf{P}}-Y_{\mathbf{H}}$. Say $x \in A$ and $\langle A\rangle \in \delta \mathbf{P}-\delta \mathbf{P}(x)$, where $A$ is a finite subset of $\mathbf{P}$ and $\langle A\rangle$ is the isomorphism class of $A$. For any subgraph $\mathbf{G}$ of $\mathbf{H}$, set $K_{\mathbf{G}}=\{x \in G:\langle A\rangle \notin \mathbf{G}(x)\}$. Note that $x \notin K_{\mathbf{H}}$ and $K_{\mathbf{H}} \varsubsetneqq A$. For each $y \in A-K_{\mathbf{H}}$, choose $A_{y} \subset H(y)$ such that $A_{y} \simeq A$. Let $\mathbf{B}=A \cup\left\{A_{y}: y \in A-K_{\mathbf{H}}\right\}$. Then $\langle B\rangle \in \delta \mathbf{H}-\delta \mathbf{P}: K_{\mathbf{B}}=K_{\mathbf{H}}$, 
but if $\mathbf{B}^{\prime}$ is a subgraph of $\mathbf{P}$ such that $\langle A\rangle \in \delta \mathbf{B}^{\prime}$, then $x \in \mathbf{B}^{\prime}$ and $K_{\mathbf{H}} \subset \mathbf{B}^{\prime}$; thus $K_{\mathbf{B}} \cup\{x\} \subset K_{\mathbf{B}^{\prime}}$, and $\left|K_{\mathbf{B}}\right|<\left|K_{\mathbf{B}^{\prime}}\right|$, so $\mathbf{B}^{\prime} \notin\langle\mathbf{B}\rangle$.

For (2), suppose that $\tau \in \delta \mathbf{H}-\delta \mathbf{P}$. Let $\mathbf{P}^{\prime}$ be a minimal extension of $\mathbf{P}$ such that $\tau \in \delta \mathbf{P}^{\prime}$. Clearly, each element of $P^{\prime}-P$ is in $Y_{\mathbf{P}^{\prime}}$, and since $Y_{\mathbf{H}} \subset \mathbf{P}$, it follows that $Y_{\mathbf{P}^{\prime}} \neq Y_{\mathbf{H}}$. Obviously, $\delta \mathbf{P}^{\prime} \neq \delta \mathbf{P}$.

To complete the proof of the lemma, consider a $k$-tuple $\bar{x}$ in $H, k<n$.

Case 1. $Y_{\mathbf{H}}-\bar{x} \neq \varnothing$. Say $y \in Y_{\mathbf{H}}-\bar{x}$. Now $\mathbf{H}$ obviously has a subgraph $\mathbf{H}^{\prime}$ of cardinality $\mu$ such that $\delta \mathbf{H}^{\prime}=\delta \mathbf{H}$, but no subgraph of $\mathbf{H}(y)$ can be isomorphic to $\mathbf{H}^{\prime}$. Hence $\left|\mathbf{I}_{\mu} \mathbf{H}(y)\right|<N$, and by the induction on $N$ and Lemma 10(a), $d_{\mathbf{H}}(\bar{x}) \leq d_{\mathbf{H}(y)}(\bar{x})+1<N$.

Case 2. $Y_{\mathbf{H}} \subset \bar{x}$. We first show that $\left|\mathbf{I}_{\mu}\left(\mathbf{H} / Y_{\mathbf{H}}\right)\right| \leq N$. Suppose that $\mathbf{P}^{*}$ and $\mathbf{Q}^{*}$ are induced subgraphs of $\mathbf{H} / Y_{\mathbf{H}}$ of cardinality $\mu$. Let $\mathbf{P}$ and $\mathbf{Q}$ be subgraphs of $\mathbf{H}$ induced by $\mathbf{P}^{*} \cup Y_{\mathbf{H}}$ and $\mathbf{Q}^{*} \cup Y_{\mathbf{H}}$ respectively. It suffices to show that $\mathbf{P}^{*} \simeq \mathbf{Q}^{*}$ if $\mathbf{P} \simeq \mathbf{Q}$.

Suppose that $\psi$ is an isomorphism from $P$ onto $Q$. Since $Y_{\mathbf{H}} \subset P$ and $Y_{\mathbf{H}} \subset Q$, by the claim, $Y_{\mathbf{P}}=Y_{\mathbf{H}}=Y_{\mathbf{Q}}$. Thus $\psi\left[Y_{\mathbf{H}}\right]=Y_{\mathbf{H}}$ and $\psi\left\lceil P^{*}\right.$ is an isomorphism from $P^{*}$ onto $Q^{*}$.

Now by induction on $n$ and Lemma $10(\mathrm{~b}), d_{\mathbf{H}}(\bar{x})=d_{\mathbf{G}}\left(\bar{x}-Y_{\mathbf{H}}\right)<N$, where $G=\mathbf{H} / Y_{\mathbf{H}}$.

Thus we have shown that $d(\bar{x})<\left|\mathbf{I}_{\mu}(\mathbf{H})\right|$ for all $k$-tuples $\bar{x}, 0 \leq k<n$. To show that $\mathbf{S}(\mathbf{H})$ has finitely many edges, we again consider two cases:

Case 1. $\left|Y_{\mathrm{H}}\right|>n$. Let $Y$ be a set of $n+1$ elements of $Y_{\mathbf{H}}$. Then every $k$-tuple, $0 \leq k \leq n$, is in $\mathbf{H}(y)$ for some $y \in Y$. So, by Lemma 10(a), $\widetilde{E}_{\mathbf{H}} \subset \bigcup\left\{\widetilde{E}_{\mathbf{H}(y)}: y \in Y\right\}$ and we use the induction on $N=\left|\mathbf{I}_{\mu}(\mathbf{H})\right|$.

Case 2. $\left|Y_{\mathbf{H}}\right| \leq n$. Let $Y=Y_{\mathbf{H}}$. Then

$$
\widetilde{E}_{\mathbf{H}} \subset\left(\bigcup\left\{\widetilde{E}_{\mathbf{H}(y)}: y \in Y\right\}\right) \cup\left(\left\{\bar{x} \cup Y: \bar{x} \in \widetilde{E}_{\mathbf{H} / Y}\right\}\right)
$$

by Lemma 10(a) and (b), and we use the induction on $n$ and $N$.

Recall that $F_{\mathbf{H}}=\bigcup \widetilde{E}_{\mathbf{H}}$. We end this section by noting:

Corollary 3. Let $\mathbf{H}$ be an infinite n-uniform hypergraph with $\left|\mathbf{I}_{\mu}(\mathbf{H})\right|=N<\omega$.

(a) $\left|F_{\mathbf{H}}\right| \leq n(N-1)$.

(b) If $Y_{\mathbf{H}}$ is as in the proof of Lemma 9, then $Y_{\mathbf{H}}=F_{\mathbf{H}}$.

Proof. (a) Let $F^{0}=F_{\mathrm{H}}$. Suppose we have defined $F^{i}$ for all $i<j$. Choose $x \in F^{j-1}$ which is in a few edges of $\widetilde{E}_{\mathbf{H}}\left\lceil F^{j-1}\right.$ as possible. Let $S=\left\{y \in F^{j-1}\right.$ : for all $e \in \widetilde{E}_{\mathbf{H}}\left[F^{j-1}\right.$, if $y \in e$ then $\left.x \in e\right\}$, and $F^{j}=F^{j-1}-S$. Note that $|S| \leq n$. Since $\mathbf{H}$ is polarized and $\left|\mathbf{I}_{\mu}(\mathbf{H})\right|=N$, there are at most $N$ distinct $F^{i}$, one of which is $\varnothing$. Thus $\left|F_{\mathrm{H}}\right| \leq n(N-1)$.

(b) If $F_{\mathbf{H}} \subset \mathbf{H}(x)$, then $\mathbf{H} \simeq \mathbf{H}(x)$ by Theorem 7(4), and so $\delta \mathbf{H}=\delta \mathbf{H}(x)$. Thus $Y_{\mathbf{H}} \subset F_{\mathbf{H}}$. Conversely, by Lemma 6, there is a finite subgraph $\mathbf{G}$ of 
$\mathbf{H}$ containing $F_{\mathbf{H}}$, large enough so that $\mathbf{S}(\mathbf{G})=\left(G, \widetilde{E}_{\mathbf{H}}\right)$. But if $x \in F_{\mathbf{H}}$, no subgraph of $\mathbf{H}(x)$ can have signature isomorphic to $\left(G, \widetilde{E}_{\mathbf{H}}\right)$.

\section{A CLASS OF GRAPHS WITH LARGE $\mathbf{I}(\mathbf{G})$}

In this section we show that the classical examples of graphs of regular cardinality $\kappa$ with no cliques or independent subsets of cardinality $\kappa$ also have the largest possible value for $\mathbf{I}(\mathbf{G})$. These are the comparability graphs of partial orders that are the intersection of two linear orders on the same set of cardinality $\kappa$, one the type $\kappa$ well-ordering, the other an order with no suborder of type $\kappa$ or its dual. We also show that the comparability graph of a tree of cardinality $\kappa$ with no cliques or independent subsets of cardinality $\kappa$ has $|\mathbf{I}(\mathbf{G})| \geq 2^{<\kappa}$.

Definition 13. A linearly ordered set $(H, R)$ is strongly dense if it does not have end points and for all $x, y \in H$ there are $|H|$ many points between $x$ and $y$.

Lemma 12. If a linearly ordered set $(H, R)$ has no subset order-isomorphic to $\kappa$ or $\kappa^{*}$ where $|H|=\kappa$, and $\kappa$ is regular, then there is a subset $H^{\prime}$ of $H$ such that $\left|H^{\prime}\right|=\kappa$ and $\left(H^{\prime}, R\right)$ is strongly dense.

Proof. Let $x \equiv y$ if there are fewer than $\kappa$ elements between $x$ and $y$. This is an equivalence relation, and the equivalence classes are of cardinality $<\kappa$. [Regularity of $\kappa$ is needed here.] If we let $H^{\prime}$ be a subset that meets each equivalence class in one element (except the least and greatest, if such exist) then $H^{\prime}$ is as required.

We do not require $\kappa$ to be regular in

Theorem 10. Let $W$ be an order of type $\kappa \geq \omega$ on a set $H, R$ a strongly dense order on $H$, and $P=W \cap R$. Then the comparability graph $\mathbf{H}=(H, E)$ of $\mathbf{P}=(H, P)$ has $|\mathbf{I}(\mathbf{H})|=2^{\kappa}$.

Proof. We first show that we can recover $\mathbf{P}$ from $\mathbf{H}$. Indeed, $x P y$ iff both $x E y$ and $|\{z: x E z \wedge \neg y E z\}|=\kappa$ : if $x P y$ then there are $\kappa$-many $z$ between $x$ and $y$ in $\mathbf{R}=(H, R)$ and almost all of these are greater than $x$ and $y$ in $\mathbf{W}=(H, W)$; if $\neg x P y$ but $x E y$, then $y P x$, and for almost all $z$ satisfying $x E z$ we have $x P z$ and thus $y E z$.

Next we show that we can recover $W$ and $R$ from $P$. Clearly it is enough to recover $R$. Now $x R y$ iff $|\{z: x P z \wedge \neg y E z\}|=\kappa:$ if $x R y$ then almost all elements between $x$ and $y$ in $\mathbf{R}$ are greater than $y$ in $W$; if $y R x$, then for almost all $z$ satisfying $x P z$, we have $y P z$ and thus $y E z$.

Finally, we show that for every $X \subset \kappa$ there exists $H_{X} \subset H$ such that

(i) $\mathbf{R}\left\lceil H_{X}\right.$ is strongly dense and

(ii) if $\gamma$ is zero or a limit ordinal, and $n \in \omega$, then

$$
\gamma+n \in X \quad \text { iff } h_{\gamma+2 n} R h_{\gamma+2 n+1},
$$

where $h_{\alpha}$ is the $\alpha$ th element of $\mathbf{W}\left\lceil H_{X}\right.$. 
From (i) and (ii) it follows that $\mathbf{H}\left\lceil H_{X} \simeq \mathbf{H}\left[H_{Y}\right.\right.$ iff $X=Y$ : since both structures are intersections of cardinal order types with strongly dense order types, we can recover these orders and then recover $X$ and $Y$.

Construction of $H_{X}$. We construct $H_{X}$ in stages $0 \leq \alpha<\kappa$. Let $H^{\alpha}$ be the part of $H_{X}$ constructed before the $\alpha$ th stage, let $\bar{H}^{\alpha}=H^{\alpha} \cup\{-\infty, \infty]$, and let $\bar{H}=H \cup\{-\infty, \infty\}$. Let $g: \kappa \rightarrow \bar{H} \times \bar{H}$ be a surjection such that $\left|g^{-1}(a, b)\right|=\kappa$ for all $a, b \in \bar{H}$.

Stage $\alpha=\gamma+2 n$, where $\gamma$ is a limit ordinal: Choose $s \in H$ such that $s$ is $W$-greater than any element of $H^{\alpha}$ and if $g(\gamma+n)=(a, b) \in \bar{H}^{\alpha} \times \bar{H}^{\alpha}$, $a \neq b$, then $s$ is $R$-between $a$ and $b$. Let $H^{\alpha+1}=H^{\alpha} \cup\{s\}$.

Stage $\alpha=\gamma+2 n+1$, where $\gamma$ is a limit ordinal. Choose $s \in H$ such that $s$ is $W$-greater than all elements of $H^{\alpha}$ and $r R s$ iff $\gamma+n \in X$, where $r$ is the $(\gamma+2 n)$ th element of $H^{\alpha}$. Let $H^{\alpha+1}=H^{\alpha} \cup\{s\}$.

Corollary 4. Let $W$ be an order of type $\kappa$ on a set $H$ of regular infinite cardinality $\kappa$. Let $R$ be a linear order on $H$ such that $(H, R)$ has no subset order-isomorphic to $\kappa$ or $\kappa^{*}$. Then the comparability graph $\mathbf{H}$ of $(H, W \cap R)$ has $|\mathbf{I}(\mathbf{H})|=2^{\kappa}$.

In a similar but even simpler manner, any tree in which each nonmaximal element has at least two immediate successors can be recovered from its comparability graph: $x \subseteq y$ iff $\{z: x E z\} \supset\{z: y E z\}$. The comparability graph of a tree of cardinality $\kappa$ either has a clique of cardinality $\kappa$ or an independent subset of cardinality $\kappa$, or the tree is $\kappa$-Souslin: every chain and antichain is of cardinality $<\kappa$. There are degenerate examples of $\kappa$-Souslin trees for singular cardinals $\kappa$, but even in those models where there are examples for $\kappa$ regular, we have

Theorem 11. If there is a $\kappa$-Souslin tree and $\kappa$ is regular, every such tree has at least $2^{<\kappa}\left(=\sup \left\{2^{\lambda}: \lambda<\kappa\right\}\right)$ nonisomorphic subtrees of cardinality $\kappa$. Its comparability graph has at least $2^{<\kappa}$ nonisomorphic induced subgraphs of cardinality $\kappa$. In particular, every Souslin tree has at least $2^{\omega}$ nonisomorphic subtrees of cardinality $\omega_{1}$.

Proof. As is well known, every $\kappa$-Souslin tree has a subtree of cardinality $\kappa$ in which every element has at least two immediate successors and successors on all levels of the tree. So it suffices to show that such a "pruned" $\kappa$-Souslin tree has at least $2^{<\kappa}$ nonisomorphic subtrees of cardinality $\kappa$.

For each $A \in[\kappa]^{<\kappa}$, choose an element $x$ on level $(\sup A)+1$ and then, for each $\alpha \in A$, choose an immediate successor $y_{\alpha}$ of the predecessor $x_{\alpha}$ of $x$ on level $\alpha$, such that $y_{\alpha} \neq x_{\alpha+1}$, and remove all successors of $y_{\alpha}$. The resultant subtree has the property that every nonmaximal element has at least two immediate successors. Obviously, if $A \neq B$, the subtrees obtained from $A$ and $B$ cannot be isomorphic. Thus by the remark preceding the theorem these subtrees do not have isomorphic comparability graphs. 


\section{REFERENCES}

1. J. Baumgartner, Order types of real numbers and other uncountable orderings, Ordered Sets (Ivan Rival, ed.), NATO Advanced Study Institute Series C, vol. 83, Reidel, 1982, pp. 239277.

2. C. C. Chang and H. J. Keisler, Model theory, North-Holland, Amsterdam, 1973.

3. R. Jamison, private communication.

4. M. Pouzet, private communication.

Department of Mathematics, University of South Carolina, Columbia, South CarOLINA 29208 